Relations industrielles

Industrial Relations

\title{
Droits de la direction et égalité des parties
}

\section{Roger Chartier}

Volume 13, numéro 4, octobre 1958

URI : https://id.erudit.org/iderudit/1022414ar

DOI : https://doi.org/10.7202/1022414ar

Aller au sommaire du numéro

Éditeur(s)

Département des relations industrielles de l'Université Laval

ISSN

0034-379X (imprimé)

1703-8138 (numérique)

Découvrir la revue

Citer ce document

Chartier, R. (1958). Droits de la direction et égalité des parties. Relations industrielles / Industrial Relations, 13(4), 446-451.

https://doi.org/10.7202/1022414ar

Tous droits réservés @ C Département des relations industrielles de l’Université Laval, 1958
Ce document est protégé par la loi sur le droit d'auteur. L’utilisation des services d'Érudit (y compris la reproduction) est assujettie à sa politique d'utilisation que vous pouvez consulter en ligne.

https://apropos.erudit.org/fr/usagers/politique-dutilisation/ 


\title{
JURISPRUDENCE DU TRAVAIL
}

\section{Droits de la direction et égalité des parties}

\section{Roger Chartien (avec la collaboration de Me Marcel Crête, co-signataire, et la dissidence de M. Ivan Legault)}

\begin{abstract}
Une tâche nouvelle se crée à la Shawinigan Chemicals Limited. La direction lui assigne un taux horaire auquel le Syndicat national des travailleurs en produits chimiques de Shawinigan Falls, Inc. s'oppose en logeant grief, prétendant qu'il est trop bas. De part et d'autre, la preuve parâ̂t ÉGALEMENT valable et bien étoffée au président du conseil d'arbitrage. En pareil cas, comment sortir du dilemme quand on postule l'égalité réelle des parties? Aurait-il mieux valu n'en pas sortir?
\end{abstract}

A. Preuve et argumentation des parties

1. Position du Syndicat

Il s'agit ici d'une nouvelle tâche, accomplie dans une usine d'acide sulphurique nouvellement ouverte à Shawinigan-Est. C'est donc l'article 405 qui s'applique:

Les taux horaires de paie applicables aux nouvelles occupations ou aux occupations existantes qui sont substantiellement changées et qui peuvent survenir pendant la durée de cette convention, seront déterminés par la gérance qui tiendra compte des taux des emplois existants de nature similaire.

Cependant, après une période de quatre-vingt-dix (90) jours, !es employés qui croient ne pas être traités équitablement ont le droit d'en appeler tel que prévu à l'article IX (procédure des griefs).

Le Syndicat se prévaut de cette clause pour loger un grief relatif au taux de lopérateur employé dans cette nouvelle usine.

Le procureur syndical déclare que ce taux est le même que celui de l'assistant «boiler operator» qui détient une licence 4A dans les divisions du Carbure et des Produits chimiques. Or, les possesseurs de ce dernier titre font le même travail, quelle que soit leur licence et même avec ou sans licence. Ils n'ont pas la responsabilité de l'opération d'une bouilloire et sont sous les ordres directs d'autres employés qui eux sont responsables. «A Shawinigan-Est, au contraire, l'opérateur est directement responsable de l'opération de la bouilloire et des turbines 》 ( S-2, page 4, paragraphe 8). Il devrait donc gagner davantage que l'assistant-opérateur de bouilloire, de conclure le Syndicat. 
Dans les autres divisions, continue le procureur syndical, le salaire horaire minimum d'un opérateur responsable de l'opération d'une bouilloire ( «boiler operator - 1st fireman - holding $3 \mathrm{~A} \gg$ ) est de $12 \phi$ plus élevé que le salaire de l'opérateur ici en cause. Etant donné la différence entre $3 \mathrm{~A}$ et $4 \mathrm{AB}$, le Syndicat s'estime donc justifié de demander $6 \phi$ d'augmentation en faveur de ce dernier, avec rétroactivité à la date à laquelle il a assumé sa fonction.

Après une série de comparaisons du même ordre d'une division à l'autre, le procureur syndical termine en invoquant plusieurs articles et paragraphes de la «Loi concernant les mécaniciens de machines fixes» (particulièrement la section III, paragraphes 9 et 10 ) et des «Règlements 》 y reliés.

\section{Position de la Compagnie}

Le témoin de la Compagnie est Andrew C. Holm, surintendant, depuis le ler janvier 1957, de la division de Shawinigan-Est de la Compagnie. Il est l'auteur de la pièce C-1-o, épine dorsale de la preuve patronale, qu'il vient expliciter et défendre devant ce conseil d'arbitrage.

Normalement, selon lui, le taux de l'opérateur en question devrait être de $\$ 1.95$ (au ler août 1957), d'après la cédule « $\mathrm{A}$ » de la convention, page 42, «Operation - Chemical », pour la tâche intituı!ée «All operators not otherwise specified ». Admettant l'argument syndical de la responsabilité de cet opérateur à l'égard d'une bouilloire (et d'une turbine), il estime raisonnable et suffisant qu'une «prime» de $8 \phi$ l'heure vienne récompenser cette responsabilité.

D'autre part, il s'oppose à la comparaison que propose le Syndicat entre l'opérateur en cause et les mécaniciens de machines fixes des deux autres divisions (Carbure et Produits chimiques), déjà couverts par la cédule «A », qui fut établie bien avant la création de la nouvelle tâche et sans en tenir compte, évidemment. De plus, l'opérateur de Shawinigan-Est ne passe que le quart de son temps autour de la bouilloire (et le reste autour du processus chimique lui-même), tandis que ceux auxquels on veut le comparer y passent tout leur temps. Et encore, les bouilloires des deux autres divisions ( 11 en tout) sont plus grosses, plus compliquées, et donc exigent de leurs opérateurs plus d'habileté et d'expérience que la petite bouilloire de Shawinigan-Est. «The Boiler Code requires at least 5 years practical boiler experience before an operator could lawfully assume responsibility for the Carbide or Chemical boilers (with a $2 \mathrm{~A}$ paper). At the sulphuric acid plant, the corresponding qualifying period would be $11 / 2$ years (for a $4 \mathrm{AB}$ paper) 》, d'affirmer Holm dans son mémoire (C-1-o, p. 2, c.).

Tous les «assistants 》 des opérateurs de bouilloires, quelle que soit leur classe (4A, 3A, 2A), exécutent le même travail, et donc n'ont pas de responsabilités différenciées. Mais aux deux autres divisions, pour les encourager à se perfectionner en prévision d'une promotion éventuelle à des tâches plus qualifiées, la Compagnie a mis en pratique le paiement de «primes »: c'est ainsi que la licence $4 \mathrm{~A}$ vaut $7_{\phi}$ de plus l'heure que l'absence de licence, et la licence $3 \mathrm{~A}, 5 \phi$ de plus que la licence $4 \mathrm{~A}$. Un tel système ne se justifie pas à Shawinigan-Est, puisqu'il n'existe qu'une seule occupation relative à la bouilloire. 
En somme, de dire Holm, le Syndicat demande deux «primes », dont seule la première, fondée sur la responsabilité, est justifiée, et d'ailleurs récompensée par la Compagnie. D'autre part, cette responsabilité est assez limitée, puisque c'est une sonnette d'alarme qui avertit l'opérateur d'une baisse anormale du niveau de l'eau, et que si des bris surviennent, d'autres mécaniciens sont immédiatement appelés.

Enfin, si la Compagnie était contrainte de donner suite à la demande syndicale, il s'ensuivrait des «anomalies sérieuses» dans la structure des salaires qui donneraient occasion à de nouveaux litiges: le mémoire Holm donne trois exemples précis de ces «anomalies».

Le grief du Syndicat, aux yeux de la Compagnie, doit donc être renvové.

\section{B. Commentaires des arbitres}

Comment trancher pareil litige? Les arbitres se trouvent en présence d'une preuve bien étoffée de part et d'autre, de prétentions syndicales sensées auxquelles s'oppose un raisonnement patronal au moins également raisonnable. Faudrait-il, à l'instar de Salomon mais allant jusqu'au bout de son geste seulement esquissé, donner raison à demi au Syndicat et à moitié à la Compagnip? Cette forme facile de maquignonnage n'aurait vraiment pas réglé le problème. Ce conseil d'arbitrage a donc décidé d'exposer clairement les «principes » qui l'ont inspiré dans sa décision.

\section{Egalité réelle des parties}

Il faut solidement établir, comme premier postulat, que les parties (Syndicat et Compagnie) qui se présentent devant un conseil d'arbitrage sont vraiment égales entre elles, d'une éga'ité beaucoup plus précise et moins théorique ou nébuleuse que celle qui préside à l'élaboration de formules comme: «Tous les hommes naissent égaux», ou «Tous sont égaux devant la loi ». Cette égalité, qui est source de liberté et condition essentielle de toute relation contractuelle valide, constitue l'assise même et l'objectif constant de notre législation du travail et de la négociation collective qui en est le coeur. Et l'Etat, à la suite du législateur, veille ou doit veiller au maintien de cette égalité.

Si d'une part la Compagnie fournit le capital, les bâtiments, l'outillage et les matières nécessaires, de l'autre le Syndicat fournit le personnel. L'un étant absolıment nécessaire aux autres, et vice versa, un acoord entre égaux devient nécescaire, dont des négociations plus ou moins dures établiront les clauses.

Selon nous, l'arbitre et surtout le président qui n'admettrait pas ce postulat pourrait faire des dégâts considérables, et de toutes façons n'aurait pas longue carrière.

\section{Les droits de la direction}

Mais alors, comment expliquer ces fameux «droits de gérance », ces prérogatives directoriales ou patronales que l'on trouve définis dans la plupart des conventions collectives d'aujourd'hui? 
Not only does management have the general right to manage the business, but many agreements provide that management has the exclusive right to direct working forces and usually to lay off, recall, discharge, hire, etc.

The right to direct... does not imply some right over and above labor's right. It is a recognition of the fact that somebody must be boss...

Management decides what the employee is to do. However, this right to direct or to initiate action does not imply a second-class role for the union. The union has the right to pursue its role of representing the interest of the employee with the same stature accorded it as is accorded management... The company direots and the union grieves when it objects... It is essential that arbitrators not give greater weight to the directing force than the objecting force... [so that] grievance [have to] be extra well-founded to justify interference with this right to manage.

(Arthur J. Goldberg. «Management's Reserved Rights: A Labor View », dans Management Rights and the Arbitration Process (Jean T. McKelvey, ed.) Washington, D.C.: BNA Incorp., 1956, pp. 119ff.)

Goldberg, qui est le principal conseiller juridique des United Steelworkers of America, reconnaît donc cette nécessité d'une direction qui... dirige, de même que la légitimité des clauses dites de «droits de gérance»dans les conventions collectives. Il n'insiste pas moins sur le fait de l'égalité fondamentale des parties, même si l'une doit prendre l'initiative de décisions que l'autre exécutera, ou refusera d'exécuter en logeant grief. Là toutefois où il pose un problème crucial aux arbitres, c'est quand il leur demande, au nom même de cette égalité des parties, de ne pas exiger du Syndicat que ses griefs soient parfaitement bien fondés et justifiés ( extra well-founded 》) avant de se prononcer, le cas échéant, contre la position patronale.

Commentant la position de Goldberg dans le même ouvrage, le professeur Neil W. Chamberlain, grand spécialiste des relations entre syndicat et direction aux Etats-Unis et auteur réputé, jette une lumière utile sur ce difficile aspect de la question:

There is a sense in which management must be given preferential treatment because it is the initiator, if we are to have any rationality to organizational life. The man who has not only the power but also the duty of initiating action must be given the right of reasonable judgment. If... a member of management makes his decision and that decision is reasonable under the circumstances and under the terms of that agreement, that decision must be honored even though the union argues that he should have taken another course of action which might also be viewed as reasonable... Management requires initiative, and initiative requires discretion and exercise of judgment, and if that judgment is exercized fairly, it could be upheld even though the union - equally fairly would have it otherwise...

(pp. 139-140)

Nous nous rallions, comme arbitres, à cette dernière formulation. Si les parties vivaient sous un régime de co-gestion dans les matières qui nous occupent, la perspective serait tout autre, et un différend comme celui-ci se règlerait vraisemb'ablement à sa source même, en pourparlers directs, sans atteindre le niveau de l'arbitrage. Mais il ne s'agit pas de cela ici. La direction a par définition le droit 
de diriger la main-d'oeuvre; plus, elle a le devoir de le faire. On attend d'elle qu'elle amorce des ordres, qu'elle prenne des décisions. S'il n'y avait pas de convention co'lective, ces ordres seraient sans réplique, ces décisions, finales, compte tenu du seul droit commun.

Mais la convention collective inclut presque toujours une clause de «droits de gérance » d'un type spécial qui, tout en reconnaissant certaines prérogatives patronales, soumet ces dernières à des formes plus ou moins sévères de contrôle syndical. L'article II de la convention collective qui nous intéresse plus particulièrement ici vaut d'être cité comme exemple:

\section{FONCTION DE LA GÉRANCE.}

200 - Le Syndicat accepte que la gérance a le droit exclusif de prendre toute décision relative aux fonctions de la gérance et à la direction générale de l'usine, notamment:... [suivent des points précis].

201 - Rien dans cet article ne doit être interprété comme annulant toute autre stipulation de la présente convention. Cet article n'a pas priorité sur les autres stipulations de la convention mais leur est subordonné; il accorde à la gérance le droit d'agir mais ne donne pas à ses décisions un caractère irrévocable devant un Conseil d'arbitrage.

Nous sommes donc d'accord, en théorie et en pratique: le Syndicat «accorde à la gérance le droit d'agir $»$, donc respecte son initiative et ne nuit pas à l'amorce de ses décisions, tout en refusant à celles-ci «un caractère irrévocable devant un Conseil d'arbitragè ».

Mais quand ces décisions seront-elles révocables par des arbitres? Toute la question est là. Avec Chamberlain, nous estimons que si l'organisation industrielle d'aujourd'hui doit maintenir une certaine unité interne, garder un certain ordre et ne pas s'effriter dans l'anarchie la plus complète, il est nécessaire que les conseils d'arbitrage accordent comme un traitement de faveur ( à la position patronale par rapport à la position syndicale. Et avant d'entendre les hauts cris que justifierait notre position «égalitaire» du début, nous nous hâtons de nous expliquer.

Quand un membre de la direction pose un geste ou prend une décision (en vertu des droits de gérance) qui, après étude sérieuse, semblent raisonnables aux arbitres, et que par la suite, le Syndicat (en vertu de son droit de grief) propose une solution qui, dans les circonstances et d'après les stipulations de la convention, paraît ÉGALEMENT raisonnable aux mêmes arbitres, ceux-ci n'ont pas d'autre recours que de donner le «bénéfice du doute » à celui dont c'est la responsabilité d'amorcer des décisions.

Si d'autre part la position syndicale (présentée en vertu de la procédure des griefs) apparaît aux arbitres comme plus sensée et plus solide que la position patronale, i's donneront sans doute raison au Syndicat. Celui-ci doit savoir, cependant, ce qui trotte dans la tête des arbitres quand des questions de ce genre se présentent à eux, et comprendre combien ces griefs doivent être bien fondés et solidement défendus s'il veut l'emporter à leurs yeux.

Pour être encore plus précis, donnons quelques exemples. Qu'il s'agisse de notation du personnel (merit rating) plus ou moins systématisée (où un employé 
s'estime justifié de monter à l'intérieur ou au maximum d'un « range » de salaire donné), de reclassification ascendante ou de promotion (qu'un travailleur croit mériter), nous estimons qu'en l'absence de stipulations contractuelles précises, les décisions patronales mises en question par le Syndicat ne seront renversées par les arbitres que si le Syndicat peut démontrer avec précision et clarté que la Compagnie, en l'occurrence, a fait preuve d'incompétence, d'inconséquence, d'incurie ou d'ignorance, et fait montre de malhonnêteté, d'injustice, de mauvaise foi et de «discrimination» au sens anglais du mot, i.e. si elle a utilisé des critères non pertinents pour en arriver à ses décisions (s'en remettant aux charmes, aux animosités personnelles, à la race, la couleur, la religion ou la nationalité au lieu de se référer aux mérites professionnels de ses employés). En dehors de ces cas, les arbitres pourraient avoir certaine difficulté à s'inscrire en faux contre les décisions de la direction.

Et ce qui rassure temporairement ce conseil d'arbitrage sur son orthodoxie, c'est la formulation même des articles 405 et 406 de la convention collective, qui ont servi d'amorce à ce grief et dont nous citons et soulignons quelques passages:

405 - Les taux horaires de paie applicables aux nouvelles occupations... seront déterminés par la gérance... Les employés qui croient ne pas être traités équitablement ont le droit d'en appeler...

406 - Les employés qui sont payés moins que le taux maximum et croient ne pas être traités équitablement ont le droit d'en appeler...

\section{DÉCISION}

Fidèle aux principes qu'il vient d'énumérer, ce conseil d'arbitrage, conscient de la qua'ité de la preuve syndicale mais aussi très favorablement impressionné par la preuve patronale, DÉCIDE À LA MAJORITÉ, l'arbitre syndical étant dissident, qu'il n'y a eu ni incurie, ni incompétence, ni injustice, ni mauvaise foi de démontrées de la part de la Compagnie dans la fixation du taux de l'opérateur de Shawinigan-Est, et donc que ce grief doit être renvoyé.

\section{Dissidence de M. Ivan Legault, arbitre syndical}

Nous ne pouvons partager l'opinion de nos collègues à l'effet que devant une preuve de valeur égale de part et d'autre, le bénéfice du doute doive être accordé à la Compagnie en raison de la responsabilité qui lui échoit d'amorcer des décisions.

Des nuances s'imposent. Nous admettrions plus volontiers leur point de vue s'il s'agissait d'un différend portant par exemple sur la détermination du standard de la qualité à produire, le choix des fournisseurs ou des produits à fabriquer; ce qui ressort en général du domaine strictement technique de l'entreprise. Mais nous croyons qu'il en va autrement lorsque, comme dans le cas présent, c'est la fixation du taux de rémunération d'une occupation donnée qui est en litige. 